\title{
MIGRACIONES INTERURBANAS EN LA FRONTERA NORTE DE MÉXICO
}

\author{
por \\ Gabriel Estrella Valenzuela*
}

\begin{abstract}
RESUMEN
Este ensayo describe la migración de las últimas cuatro décadas hacia los estados fronterizos del norte de México en general, y hacia Baja California en particular. Para poder comparar los saldos migratorios fronterizos se establece una subregionalización de los estados y municipios adyacentes a la frontera.

El fenómeno migratorio de Baja California se aborda a partir del perfil de los movimientos poblacionales en cuanto a magnitud, origen y destino. Además, se analizan las características de los migrantes intraestatales que residen en las áreas urbanas de Baja Califonia. Para finalizar, se presentan las conclusiones del trabajo.
\end{abstract}

\begin{abstract}
This essay describes the migration held during the last four decades towards the border states of the north part of Mexico in general, and towards Baja California in particular. In order to compare the frontier migratory settlements, the author establishes a subregionalization of the states and adjacent border districts.

The migratory phenomenon of Baja California is aproached from the profile of the population movements as for its magnitude, origin and destiny.

The characteristics of the inter-states migrants that reside in the urban areas of Baja California are analized as well. The conclusions of the study are presented to finalize the essay.
\end{abstract}

\section{INTRODUCCIÓN}

$\mathrm{La}$ intención central de este trabajo consiste en exponer algunas características de la migración interurbana que se desarrolla en el estado de Baja California, así como las implicaciones que se derivan de dichas características para las políticas de descentralización hacia ciudades medias.

Para lograr ese objetivo, el trabajo se ha estructurado en cuatro apartados que respectivamente abordan los temas relativos a la migración en el contexto de la frontera norte de México (FNM); la migración en Baja California; las migraciones interurbanas en Baja California, y las conclusiones de este ensayo.

* Investigador de tiempo completo en el Instituto de Investigaciones Sociales de la Universidad Autónoma de Baja California. 
En el primero de los apartados, se describen los principales rasgos que han presentado los movimientos de población hacia y en la FNM en el transcurso de las últimas cinco décadas. A continuación, en el segundo apartado, se ubica en el contexto poblacional de Baja California a las corrientes migratorias que han sido identificadas en investigaciones recientes a fin de generar un perfil de la magnitud, origen y destino de estos movimientos poblacionales.

Con este antecedente, en el tercer apartado se analizan las características de los migrantes intraestatales que residen en las áreas urbanas de Baja California, atendiendo en particular los rasgos referidos al volumen, origen, destino, temporalidad, estructura por edad y sexo, escolaridad y participación económica de los inmigrantes urbanos intermunicipales.

Por último, en el cuarto apartado se realiza una breve recapitulación de lo expuesto para derivar de ello el conjunto de conclusiones de este trabajo.

\section{LA MIGRACIÓN EN EL CONTEXTO DE LA FRONTERA NORTE DE MÉXICO ${ }^{1}$}

\section{Los estados fronterizos}

El conjunto territorial que integran los estados de Baja California, Coahuila, Chihuahua, Nuevo León, Sonora y Tamaulipas, ha sido escenario de una serie de importantes acontecimientos poblacionales en los affos transcurridos en 1940 a la fecha.

Por su magnitud y trascendencia para el área en cuestión, aquí se presentan aquellos sucesos relacionados con el volumen y ritmo de crecimiento de la población de estas seis entidades del país, con la distribución de esa población en el interior del territorio conjunto de esos estados, y con la composición de la población a partir de su condición nativa 0 inmigrante de la entidad de residencia.

Respecto al primer rubro mencionado, la información que se presenta en el cuadro 1 permite identificar varios rasgos del cambiante perfil poblacional de la frontera. El primero de ellos consiste en la magnitud del cambio observado en el total de población de los seis estados fronterizos, la cual se cuadruplicó de 1940 a 1980 al incrementarse de 2.6 millones a casi 10.7 millones de habitantes. A manera de comparación, se puede mencionar que en el mismo lapso la población total del país aumento 3.4

1 El contenido de este apartado ha sido publicado por el autor bajo el título de "Migración y Población en la Frontera Norte" en: Visión histórica de la Frontera Norte de México. Centro de Investigaciones Históricas UNAM-UABC, Mexicali, 1987. T. III, pp. (391-398). 
veces (de 19.7 a 66.9 millones de habitantes), y que debido a este menor incremento en el total nacional, la población de las entidades fronterizas logró aumentar su participación del 13.3 al $16.0 \%$ en el total de la población de México entre 1940 y 1980.

El segundo rasgo que permite identificar la misma información, es el relativo a la distribución de la población de las entidades fronterizas en el interior del área que ellas integran. Si dicha zona se subdivide para efectos de comparación en las subregiones noroeste (integrada por Baja California y Sonora) norte (integrada por Chihuahua) y noreste (integrada por Coahuila, Nuevo León y Tamaulipas), se puede observar que en 1940 estas subregiones contenían el $16.9,23.9$ y $59.2 \%$ del total de la población de la FNM, respectivamente.

Para 1980, la subregión noroeste agrupaba al $25.2 \%$ del total de la población de la FNM; la subregión norte contaba con el $18.7 \%$ de ese total y la noreste con el $56.1 \%$. De esta forma, en el transcurso de esos cuarenta años se observa una paulatina pero sostenida tendencia a la redistribución de la población en el interior de la zona fronteriza, en la que la subregión noroeste denota incrementos constantes en su participación del total poblacional, mientras que las subregiones norte y noreste acusan reducciones en su peso porcentual.

El tercer rasgo del perfil demográfico de las entidades fronterizas (contenido en la información del cuadro 1), es el relativo a la composición de la población según su condición de inmigrante o nativa de la entidad en que reside. La primer característica que se debe señalar, consiste en el hecho de que la población no nativa (o inmigrante) que reside en la FNM casi se ha quintuplicado en el transcurso de las cuatro décadas consideradas, pues su número aumentó de casi 530 mil inmigrantes en 1940 a 2.5 millones de residentes no nativos en 1980.

Por otra parte, una segunda característica que se aprecia en los datos del cuadro 3 , estriba en que la población no nativa de las entidades fronterizas ha crecido a ritmos superiores que los de la población total y nativa de esos estados, por lo menos en tres de las cuatro décadas analizadas. Este comportamiento en el ritmo de crecimiento de la población inmigrante de la FNM, ha dado por resultado el hecho de que aún en términos proporcionales su participación en el total de la población de la zona se incrementara de $20.2 \%$ en 1940 a $23.4 \%$ en 1980.

En materia de composición de la población fronteriza, el último rasgo que consideramos oportuno señalar en este apartado es aquél que nos permite observar que en el transcurso del lapso de 1940 a 1980, la distribución de la población inmigrante que reside en los seis estados considerados se ha modificado notoriamente. Al respecto, utilizando de 
CUADRO 1. Estados de la frontera norte. Población total, nativa e inmigrante (1940-1980).

\begin{tabular}{|c|c|c|c|c|c|c|c|}
\hline AÑO Y POB. & TOT. FNM & B. C. & COAH. & CHIH. & N. L. & SON. & TAMPS. \\
\hline \multicolumn{8}{|l|}{1940} \\
\hline Total & 2617723 & 78907 & 550717 & 623944 & 541147 & 364176 & 458832 \\
\hline Nativa & 2087984 & 31216 & 420499 & 531141 & 451431 & 321181 & 332516 \\
\hline Inmigrante & 529739 & 47691 & 130218 & 92803 & 89716 & 42995 & 126316 \\
\hline \multicolumn{8}{|l|}{1950} \\
\hline Total & 3762963 & 226965 & 720619 & 846414 & 740191 & 510607 & 718167 \\
\hline Nativa & 2889207 & 78389 & 569176 & 723243 & 596044 & 442671 & 479684 \\
\hline Inmigrante & 873756 & 148576 & 151443 & 123171 & 144147 & 67936 & 238483 \\
\hline \multicolumn{8}{|l|}{1960} \\
\hline Total & 5541100 & 520165 & 907734 & 1226793 & 1078848 & 783378 & 1024182 \\
\hline Nativa & 4102539 & 196426 & 743606 & 997654 & 814120 & 634919 & 715814 \\
\hline Inmigrante & 1438561 & 323739 & 164128 & 229139 & 264728 & 148459 & 308368 \\
\hline \multicolumn{8}{|l|}{1970} \\
\hline Total & 7848169 & 870421 & 1114956 & 1612525 & 1694689 & 1098720 & 1456858 \\
\hline Nativa & 6248466 & 511608 & 971612 & 1429672 & 1285242 & 930812 & 1119520 \\
\hline Inmigrante & 1599703 & 358813 & 143344 & 182853 & 409447 & 167908 & 337338 \\
\hline \multicolumn{8}{|l|}{1980} \\
\hline Total & 10691887 & 1177886 & 1557265 & 2005477 & 2513044 & 1513731 & 1924484 \\
\hline Nativa & 8191374 & 632525 & 1302100 & 1720241 & 1864286 & 1247653 & 1424569 \\
\hline Inmigrante & 2500513 & 545361 & 255165 & 285236 & 648758 & 266078 & 49915 \\
\hline
\end{tabular}

FUENTE: VI, VII, VIII y $\mathrm{X}$ Censos generales de población y vivienda. Para B.C., Coah., Chih., N.L., Son y Tamps. D.G.E. INEGI-S.P.P. 
nucvo la subregionalización aplicada para analizar la redistribución de la población total, se puede advertir que la subregión noreste disminuyó su purticipación en el total de inmigrantes residentes en los estados fronteri1 s, del $65.4 \%$ en 1940 al $56.1 \%$ en 1980 ; la subregión norte por su parte, en el mismo lapso también se redujo su participación en dicho total de 17.5 a $11.4 \%$, y mientras tanto, la subregión noroeste registró incrementos que olevaron su peso en la distribución de inmigrantes, del 17.1 al $32.5 \%$ entre 1940 y 1980.

\section{Los municipios fronterizos}

La serie de modificaciones poblacionales que se presentaron en las seis entidades de la FNM entre 1940 y 1980, se vieron acompañadas de un proceso paralelo que involucró fundamentalmente a los 36 municipios de esas entidades que colindan directamente con la frontera internacional.

En esta franja fronteriza que integran tres municipios de Baja Calfornia, seis de Coahuila, seis de Chihuahua, uno de Nuevo León, diez de Sonora y diez de Tamaulipas, se repitieron en forma acentuada las modificaciones del perfil demográfico que se presentaron en las seis entidades de la FNM de 1940 a 1980, particularmente las relacionadas con el volumen, distribución y composición de la población que reside en dicha franja.

Respecto al volumen de la población de los municipios fronterizos, se puede observar (cuadro 2) que su monto total se incrementó de 383 mil habitantes en 1940, a 2.95 millones de residentes en 1980. Este aumento de población en la franja de municipios, implicó ritmos de crecimiento que resultaron mayores que los ritmos de incremento del conjunto de las entidades en tres de las cuatro décadas consideradas como lo indican los datos del cuadro 3.

El proceso de crecimiento de la población de los municipios fronterizos se presentó acompañado de una doble redistribución de los residentes de la frontera. Por una parte, al crecer en una forma más dinámica que los estados en su conjunto, la población de los municipios de la franja aumentó su participación en el total de habitantes de las seis entidades, del $14.6 \%$ en 1940 al $27.6 \%$ en 1980 .

Por otra parte, en ese mismo lapso la población de los municipios fronterizos modificó su distribución en el interior del área integrada por éstos. Dicha modificación fue de tal magnitud que implicó la inversión de la distribución de la población en las subregiones que conforman la franja fronteriza. En este proceso, la subregión que integran los municipios del noreste ( 17 en total) redujo su participación en el total de población de la franja fronteriza, del $47.9 \%$ al $34.1 \%$ entre 1940 y 1980 ; la subregión norte, 
CUADRO 2. Municipios de la frontera norte. Total de población, nativa y no nativa por estado.

\begin{tabular}{|c|c|c|c|c|c|c|c|}
\hline \multicolumn{2}{|c|}{ AÑO Y POB. TOTAL FNM } & B. C. & СОАН. & СНIH. & N. L. & SON. & TAMPS. \\
\hline \multicolumn{8}{|l|}{1940} \\
\hline Total & 383068 & 66376 & 43651 & 84232 & 471 & 48909 & 139429 \\
\hline Nativa & 240161 & 23779 & 34867 & 54579 & 341 & 38767 & 87828 \\
\hline Inmigrante & 142907 & 42597 & 8784 & 29653 & 130 & 10142 & 51601 \\
\hline \multicolumn{8}{|l|}{1950} \\
\hline Total & 829297 & 195888 & 75353 & 170249 & 375 & 88259 & 299173 \\
\hline Nativa & 449215 & 63933 & 53438 & 109883 & 256 & 69656 & 152049 \\
\hline Inmigrante & 380082 & 131955 & 21915 & 60366 & 119 & 18603 & 147124 \\
\hline \multicolumn{8}{|l|}{1960} \\
\hline Total & 1480496 & 455231 & 90529 & 323464 & 446 & 142265 & 468561 \\
\hline Nativa & 819158 & 164174 & 75163 & 198279 & 265 & 106004 & 275273 \\
\hline Inmigrante & 661338 & 291057 & 15366 & 125185 & 181 & 36261 & 193288 \\
\hline \multicolumn{8}{|l|}{1970} \\
\hline Total & 2219130 & 754998 & 100846 & 483582 & 370 & 215136 & 664198 \\
\hline Nativa & 1533238 & 438301 & 86160 & 370782 & 257 & 168928 & 468810 \\
\hline Inmigrante & 685892 & 316697 & 14686 & 112800 & 113 & 46208 & 195388 \\
\hline \multicolumn{8}{|l|}{1980} \\
\hline Total & 2954698 & 1002461 & 142941 & 631330 & 16479 & 312087 & 849400 \\
\hline Nativa & 1921455 & 533962 & 116428 & 459824 & 11434 & 227775 & 572032 \\
\hline Inmigrante & 1033243 & 468499 & 26513 & 171506 & 5045 & 84312 & 277368 \\
\hline
\end{tabular}

FUENTE: VI, VII, VIII, $\mathrm{X}$ y $\mathrm{X}$ Censos generales de población y vivienda. D.G.E.-INEGI-SP.P. 
imtegrada por seis municipios, también disminuyó su participación porcenItual, del $22.0 \%$ al $21.4 \%$ en esas cuatro décadas, mientras que la subregión noroeste (con 13 municipios) incrementó su peso proporcional de 30.1 a 14.5\% en ese periodo de cuarenta años.

El crecimiento y la redistribución que se observaron en la población de los municipios fronterizos del norte del país, se sustentaron en la creciente Influencia que ejerció el flujo de inmigrantes en la composición de la población de esta área. Al respecto, la información del cuadro 2 permite identificar dos rasgos importantes: el primero se refiere al volumen de habitantes no nativos que residen en los municipios fronterizos, el cual se Incrementó de 143 mil en 1940, a un millón 33 mil en 1980. Este crecimiento implico que la participación de la franja fronteriza en el total de inmigrantes que habitan en los seis estados de la FNM, aumentara del $27.0 \mathrm{al} 41.3 \%$ entre 1940 y 1980 . El segundo rasgo de importancia que se deriva de la misma información, consiste en el cambio de ubicación de la población no nativa en los municipios de la franja norte; así, mientras que en 1940 la distribución de la población no nativa era de $36.9,20.8$ y $42.3 \%$ para las subregiones noroeste, norte y noreste respectivamente, para 1980 las proporciones se modificaron a $53.4,16.6$ y $30.0 \%$, en el mismo orden mencionado de las subregiones.

\section{El contexto migratorio}

A partir de lo hasta aquí expuesto, se puede afirmar que el perfil demográfico de los seis estados y 36 municipios que integran la frontera norte de México, entre 1940 y 1980 ha presentado los siguientes rasgos que redefinieron el contexto migratorio en esta zona del país:

a) La FNM ha sido receptáculo de una redistribución de la población nacional, en lo que aparece como una paulatina reubicación hacia las entidades del norte.

b) En el interior del área que abarcan estos estados fronterizos, la subregión noroeste (que integran Baja California y Sonora) ha incrementado constantemente su participación en el total de población de las seis entidades.

c) Tanto por su magnitud absoluta como por su ritmo de crecimiento, la población inmigrante que reside en estas entidades puede ser considerada como el factor predominante del aumento poblacional de los estados fronterizos.

d) La población inmigrante ha modificado su distribución en el marco territorial de los seis estados, haciendo participar en forma creciente a la subregión noroeste en el total de población no nativa. 
CUADRO 3. Estados y municipios de la frontera norte.

Tasa de crecimiento medio anual.

\begin{tabular}{|c|c|c|c|c|c|c|c|c|}
\hline \multirow{2}{*}{\multicolumn{2}{|c|}{$\begin{array}{l}\text { Década } \\
\text { Total de población }\end{array}$}} & \multirow{2}{*}{ Total } & \multirow{2}{*}{ B.C. } & \multirow[t]{2}{*}{ Coah. } & \multirow[t]{2}{*}{ Chilh. } & \multirow[t]{2}{*}{ N.L. } & \multicolumn{2}{|c|}{ Son. Tamps. } \\
\hline & & & & & & & & \\
\hline $1940^{\circ}$ & E.F. & 3.7 & 11.1 & 2.7 & 3.1 & 3.2 & 3.4 & 4.6 \\
\hline 1950 & M.F. & 8.0 & 11.4 & 5.6 & 7.3 & (2.3) & 6.1 & 7.9 \\
\hline 1950 & E.F. & 4.0 & 8.7 & 2.3 & 3.8 & 3.8 & 4.4 & 3.6 \\
\hline 1960 & M.F. & 6.0 & 8.8 & 1.9 & 6.6 & 1.8 & 4.9 & 4.6 \\
\hline 1960 & E.F. & 3.5 & 5.3 & 2.1 & 2.8 & 4.6 & 3.4 & 3.6 \\
\hline 1970 & M.F. & 4.1 & 5.2 & 1.1 & 4.1 & (1.9) & 4.2 & 3.6 \\
\hline 1970 & E.F. & 3.1 & 3.1 & 3.4 & 2.2 & 4.0 & 3.3 & 2.8 \\
\hline 1980 & M.F. & 2.9 & 2.9 & 3.6 & 2.7 & N.D. & 3.8 & 2.5 \\
\hline \multicolumn{9}{|c|}{ Población nativa } \\
\hline 1940 & E.F. & 3.3 & 9.6 & 3.1 & 3.1 & 2.8 & 3.3 & 3.7 \\
\hline 1950 & M.F. & 6.5 & 10.4 & 4.4 & 7.3 & $(2.8)$ & 6.0 & 5.6 \\
\hline 1950 & E.F. & 3.6 & 9.6 & 2.7 & 3.3 & 3.2 & 3.7 & 4.1 \\
\hline 1960 & M.F. & 6.2 & 9.9 & 3.5 & 6.1 & 0.4 & 4.3 & 6.1 \\
\hline 1960 & E.F. & 4.3 & 10.1 & 2.7 & 3.7 & 4.7 & 3.9 & 4.6 \\
\hline 1970 & M.F. & 6.5 & 10.3 & 1.4 & 6.5 & $(0.3)$ & 4.8 & 5.5 \\
\hline 1970 & E.F. & 2.7 & 2.1 & 3.0 & 1.9 & 3.8 & 3.0 & 2.4 \\
\hline 1980 & M.F. & 2.3 & 2.0 & 3.1 & 2.2 & N.D. & 3.0 & 2.0 \\
\hline \multicolumn{9}{|c|}{ Población no nativa } \\
\hline 1940 & E.F. & 5.1 & 12.0 & 1.5 & 2.9 & 4.9 & 4.7 & 6.6 \\
\hline 1950 & M.F. & 10.3 & 12.0 & 9.6 & 7.4 & $(0.9)$ & 6.3 & 11.0 \\
\hline 1950 & E.F. & 5.1 & 8.1 & 0.8 & 6.4 & 6.3 & 8.1 & 2.6 \\
\hline 1960 & M.F. & 5.7 & 8.2 & (3.5) & 7.6 & 4.3 & 6.9 & 2.8 \\
\hline 1960 & E.F. & 1.1 & 1.0 & (1.3) & $(2.2)$ & 4.5 & 1.2 & 0.9 \\
\hline 1970 & M.F. & 0.4 & 0.9 & $(0.5)$ & $(1.0)$ & $(4.6)$ & 2.5 & 0.1 \\
\hline 1970 & E.F. & 4.6 & 4.3 & 5.5 & 4.6 & 4.7 & 4.7 & 4.0 \\
\hline 1980 & M.F. & 4.2 & 4.0 & 6.1 & 4.3 & N.D. & 6.2 & 3.6 \\
\hline
\end{tabular}


e) En el mismo marco territorial, se generó una creciente concentración de la población de los estados fronterizos en los municipios que colindan con la frontera internacional.

f) En esta franja de municipios fronterizos, la redistribución de su población total favoreció la participación de la subregión noroeste de la FNM.

g) Los municipios de la subregión noroeste lograron agrupar a más de la mitad de los residentes no nativos que habitaban en los municipios fronterizos del norte de México.

\section{LA MIGRACIÓN EN BAJA CALIFORNIA}

\section{Antecedentes}

$\mathrm{Al}$ abordar el caso específico de Baja California, se deben advertir dos clementos determinantes de su particular historiografía demográfica. El primero de ellos corresponde al carácter relativamente reciente de su poblamiento, puesto que hasta 1940 aún no alcanzaba la cifra de $80 \mathrm{mil}$ habitantes. En ese momento, la entidad contaba con apenas el $3.0 \%$ de los habitantes de la FNM. Sin embargo, en el transcurso de las dos décadas siguientes, la entidad logró superar la cifra del medio millón de habitantes en un proceso de rápido crecimiento poblacional que en 1980 le permitió contar con aproximadamente 1.2 millones de personas en su territorio. De esta manera, la población de Baja California llegó a representar, en este último affo, al $11.0 \%$ del total de habitantes de la FNM.

En este intenso proceso de poblamiento de Baja California, la inmigración ha constituido el segundo elemento determinante de sus antecedentes demográficos. A nivel interno, lo anterior se pone de manifiestoal observar que la entidad contaba con 47 mil residentes no nativos en 1940, y que para 1980 la cifra se incrementa a 545 mil inmigrantes. En esta última fecha, la población no nativa aún representaba el $46.3 \%$ del total de habitantes del estado.

Considerado a nivel nacional, el proceso inmigratorio hacia Baja California adquiere relevancia al observar que, para 1980, sólo el Distrito Federal absorbió volúmenes mayores de inmigrantes absolutos que el estado. Desde esa fecha y aún en 1970, Baja California captaba aproximadamente el $5.0 \%$ del total de la inmigración absoluta nacional, lo que en consecuencia le generaba un saldo neto de migración absoluta del orden de 300 mil personas en el último año mencionado. ${ }^{2}$

2 Estimaciones realizadas con datos de: Indicadores de la Migración en México. INEGI. México, D. F. 1985. pp. 9-12. 
CUADRO 4. Población total, urbana, rural y migrante*. Baja California, 1987.

Población B.C. Total Ensenada Mexicali Tecate Tijuana

$\begin{array}{lrrrrr}\text { Total } & 1673460 & 212943 & 697519 & 39034 & 723964 \\ & & & & & \\ \text { Urbana } & 1363770 & 153685 & 489443 & 29628 & 691014 \\ \text { Rural } & 309690 & 592581 & 208076 & 9406 & 32950\end{array}$

Inmigrante

$\begin{array}{lrrrrrr}\text { Total } & 774483 & 101820 & 246413 & 22644 & 403606 \\ \text { Urbana } & 643645 & 72 & 765 & 169591 & 16938 & 384351 \\ \text { Rural } & 130838 & 29 & 055 & 76822 & 5706 & 19255\end{array}$

Emigrante

$\begin{array}{lrrrrr}\text { Total } & 47595 & 4499 & 10293 & 1303 & 31500 \\ \text { Urbana } & 38971 & 3078 & 4524 & 1090 & 30279 \\ \text { Rural } & 8624 & 1421 & 5769 & 213 & 1221\end{array}$

FUENTE: CONEPO-B.C., IS-UABC. Encuesta continua de migración de B.C. 1987.

(*) La población urbana solamente considera a los habitantes de las principales ciudades de cada municipio. La población rural corresponde al resto de los habitantes del municipio. Considera como población inmigrante a aquellos que declararon haber vivido fuera del municipio; y la población emigrante corresponde a aquella que durante los últimos 12 meses dejó la ciudad, lo cual significa 21 meses efectivos de exposición.

En este contexto estatal de alta movilidad interna de población -al que deben agregarse los flujos internacionales de población nacional y extranjera-, durante 1987 se puso en operación el proyecto denominado Encuesta Continua de Migración de Baja California ${ }^{3}$; a través de ésta, se han logrado obtener estimaciones actualizadas de los tipos y

3 Encuesta continua de Migración de Baja California, 1987. Consejo Estatal de Población de Baja Californis. Centro Regional de Investigaciones Multidisciplinarias de la Universidad Nacional Autónoma de México. Instituto de Investigaciones Sociales de la Universidad Autónoma de Baja Califomia. 
magnitudes de los movimientos de población vigentes en la entidad, los cuales se describen a continuación.

\section{Perfil actual de la migración}

\section{La emigración}

En el estudio que se menciona en el apartado anterior, se concluyó una sección especial para identificar los casos en que, en el transcurso de los 12 meses anteriores a la aplicación de la encuesta, algún miembro del hogar entrevistado hubiese cambiado su lugar de residencia de manera definitiva. Respecto a los emigrantes aś identificados, se solicitó la información relativa a relación de parentesco con el jefe del hogar, sexo, edad y lugar de residencia actual. ${ }^{4}$

Los datos correspondientes al volumen total, área y municipio de origen de los emigrantes, así como los de población total y distribución urbano-rural en los municipios, se presentan en el cuadro 4. A partir de esta información, se puede advertir que el total de emigrantes captados fue del orden de 47,600 personas. De ellas, el $81.9 \%$ emigró del medio urbano y el restante $18.1 \%$ del área rural.

Sin embargo, al considerar que el periodo de exposición fue de 21 meses efectivos, se puede estimar que el volumende emigración total por affo es de aproximadamente 27,200 personas, lo cual representa que el $1.6 \%$ de la población del estado cambia su lugar de residencia anualmente.

A nivel municipal, partiendo de la misma consideración, las tasas anuales de emigración resultan de $1.2 \%$ para Ensenada; 0.8\% para Mexicali; $1.9 \%$ para Tecate y $2.5 \%$ para Tijuana. Los tres primeros municipios mencionados aportan emigrantes en proporción menor que su participación en el total de población estatal; Tijuana, por su parte, genera el $66.2 \%$ del total de los emigrantes, evidenciando una mayor movilidad de su población que se acentúa en el caso urbano, pues este municipio genera $77.7 \%$ del total estatal de emigrantes urbanos. En la contraparte, el municipio de Mexicali concentra al $66.9 \%$ de la emigración rural de la entidad.

Una vez identificados el origen y la magnitud de los movimientos emigratorios en Baja California, los datos del cuadro 5 permiten observar la distribución de los lugares de residencia actual de quienes fueron declarados

4 Por la duración del periodo de levantamiento de la encuesta ( 9 meses), se recopilo información correspondiente a 21 meses efectivos de exposición a la migración. Las definiciones conceptuales y los procedimientos muestrales aplicados pueden ser consultados en: CONEPO-B.C., UNAM, UABC. Encuesta demográfica de Baja California. 1986. Resultados. Consejo Estatal de Población de Baja California. Mexicali, 1987. 
como emigrantes. El primer elemento que se puede mencionar al respecto consiste en que $80.8 \%$ de los movimientos corresponden a migración interna, puesto que se trata de emigraciones intramunicipales (37.3\%), intermuncipales (8.2\%) o interestatales (35.3\%). Lo anterior, sin embargo, no debe restar importancia al hecho de que casi uno de cada cinco emigrantes tenga por residencia actual a los Estados Unidos de América. En términos proporcionales, los emigrantes de Baja California que cambiaron su residencia al vecino país representaron al $18.9 \%$ del total estatal de personas que se mudaron.

Este perfil de residencia actual del total de emigrantes presenta variaciones características al ser analizado por municipios y en función de su origen urbano o rural. En el caso de los movimientos originados en las ciudades de Baja California, se puede apreciar que su estructura general coincide con la del total de emigrantes del estado; sin embargo, los emigrantes originados en Mexicali y Tecate denotan una mayor propensión a la movilidad intermunicipal, pues uno de cada cinco de ellos reside actualmente en otro municipio de la entidad (ver cuadro 6).

A su vez, la emigración total que genera el medio rural del estado presenta evidencia de tener como principal destino a otra localidad del mismo municipio (45.2\%), le siguen en orden de importancia los Estados Unidos de América (24.8\%), otro estado de México (18.9\%) y sólo al final otro municipio de Baja California. Observada a nivel municipal, la emigración rural de Ensenada se dirige mayoritariamente a otro estado del país; la de Mexicali a otra localidad municipal o a Estados Unidos; la de Tecate a otro municipio de Baja California o a los Estados Unidos, y la de Tijuana a otra localidad del mismo municipio.

Sin menospreciar la importancia evidente de la estructura de destinos antes mencionada, consideramos indispensable para los fines de este trabajo puntualizar las siguientes observaciones: a) la emigración urbana que se dirige a otro municipio de la entidad representa el $6.3 \%$ del total de emigrantes; b) el 9.3\% de aquélla se origina en la ciudad de Ensenada, Mexicali aporta el $31.9 \%$; Tecate el $7.2 \%$ y la ciudad de Tijuana contribuye con $51.5 \%$ de los emigrantes a otro municipio del estado; y c) comparativamente, la emigración urbana dirigida a otro municipio de Baja California es tres veces mayor que la originada en el área rural, lo que implica que 3 de cada 4 emigrantes que se localizan en otro municipio de la entidad proceden de alguna de las cuatro ciudades del estado.

Tomando en consideración los tres últimos elementos, a continuación se aborda el análisis de las características que presenta el grupo de población urbana que declaró haber tenido como lugar anterior de residencia a alguno de los cuatro municipios de Baja California. 
CUADRO 5. Población emigrante según origen y destino. Baja California, 1987.

\begin{tabular}{|c|c|c|c|c|c|}
\hline \multirow[b]{2}{*}{ TOTAL } & \multirow{2}{*}{$\begin{array}{l}\text { Total } \\
\text { B.C. } \\
47595\end{array}$} & \multicolumn{4}{|c|}{$\begin{array}{c}\text { MUNICIPIO DE ORIGEN } \\
\text { Ensenada Mexicali Tecate Tijuana }\end{array}$} \\
\hline & & 4499 & 10293 & 1303 & 31500 \\
\hline del mpio. & 17772 & 1427 & 4099 & 579 & 11667 \\
\hline $\begin{array}{l}\text { Otro mpio. de B.C. } \\
\text { Otro estado }\end{array}$ & 3879 & 449 & 1553 & 269 & 1608 \\
\hline de México & 16817 & 1998 & 1346 & 245 & 13228 \\
\hline Estados Unidos & 8977 & 541 & 3295 & 210 & 4931 \\
\hline $\begin{array}{l}\text { Destino no } \\
\text { especificado }\end{array}$ & 150 & 84 & 0 & 0 & 66 \\
\hline URBANAS & 38971 & 3078 & 4524 & 1090 & 30279 \\
\hline $\begin{array}{l}\text { Otra localidad } \\
\text { del mpio. }\end{array}$ & 13872 & 1002 & 1547 & 514 & 10809 \\
\hline $\begin{array}{l}\text { Otro. mpio. de B.C. } \\
\text { Otro estado de }\end{array}$ & 2993 & 279 & 956 & 216 & 1542 \\
\hline México & 15184 & 1318 & 611 & 225 & 13030 \\
\hline EstadosUnidos & 6838 & 395 & 1410 & 135 & 4898 \\
\hline $\begin{array}{l}\text { Destino no } \\
\text { especificado }\end{array}$ & 84 & 84 & 0 & 0 & 0 \\
\hline RURALES & 8624 & 1421 & 5769 & 213 & 1221 \\
\hline $\begin{array}{l}\text { Otra localidad } \\
\text { del mpio. }\end{array}$ & 3900 & 425 & 2552 & 65 & 858 \\
\hline $\begin{array}{l}\text { Otro mpio.de B.C. } \\
\text { Otro estado de }\end{array}$ & 886 & 170 & 597 & 53 & 66 \\
\hline México & 1633 & 680 & 735 & 20 & 198 \\
\hline EstadosUnidos & 2139 & 146 & 1885 & 75 & 33 \\
\hline $\begin{array}{l}\text { Destino no } \\
\text { especificado }\end{array}$ & 66 & 0 & 0 & 0 & 66 \\
\hline
\end{tabular}

FUENTE: CONEPO-BC, IS-UABC. Encuesta continua de migración de B.C. 1987.

NOTA: La población emigrante corresponde a aquélla que abandonó la localidad durante los últimos 12 meses, por lo tanto, se consideran 21 meses efectivos de exposición. 
CUADRO 6. Población emigrante según origen y destino. Baja California, 1987 (porcentajes).

\begin{tabular}{|c|c|c|c|c|c|}
\hline \multirow[b]{2}{*}{ Destino } & \multirow{2}{*}{$\begin{array}{c}\text { TOTAL } \\
\text { B.C. }\end{array}$} & \multicolumn{4}{|c|}{ MUNICIPIO DE ORIGEN } \\
\hline & & Ens. & Mxl. & Tec. & Tij. \\
\hline \multirow[t]{2}{*}{ TOTAL } & 100.0 & 9.5 & 21.6 & 2.7 & 66.2 \\
\hline & & 100.0 & 100.0 & 100.0 & 100.0 \\
\hline \multicolumn{6}{|l|}{ Otra localidad } \\
\hline del mpio. & 37.3 & 31.7 & 39.8 & 44.4 & 37.0 \\
\hline Otro mpio. de B.C. & 8.2 & 10.0 & 15.1 & 20.6 & 5.1 \\
\hline Otro estado de México & 35.3 & 44.4 & 13.1 & 18.8 & 42.0 \\
\hline Estados Unidos & 18.9 & 12.0 & 32.0 & 16.1 & 15.7 \\
\hline Destino no especificado & 0.3 & 1.9 & 0.0 & 0.0 & 0.2 \\
\hline \multirow[t]{2}{*}{ URBANAS } & 100.0 & 7.9 & 11.6 & 2.8 & 77.7 \\
\hline & 1000 & 100.0 & 100.0 & 100.0 & 100.0 \\
\hline \multicolumn{6}{|l|}{ Otra localidad } \\
\hline del mpio. & 35.6 & 32.6 & 34.2 & 47.2 & 35.7 \\
\hline Otro. mpio.de B.C. & 7.7 & 9.1 & 21.1 & 19.8 & 5.1 \\
\hline Otro estado de México & 39.0 & 42.8 & 13.5 & 20.6 & 43.0 \\
\hline Estados Unidos & 17.5 & 12.8 & 31.2 & 12.4 & 16.2 \\
\hline Destino no especificado & 0.2 & 2.7 & 0.0 & 0.0 & 0.0 \\
\hline \multirow[t]{2}{*}{ RURALES } & 100.0 & 16.5 & 66.9 & 2.5 & 14.2 \\
\hline & 100.0 & 100.0 & 100.0 & 100.0 & 100.0 \\
\hline \multicolumn{6}{|l|}{ Otra localidad } \\
\hline del mpio. & 45.2 & 29.9 & 44.2 & 30.5 & 70.3 \\
\hline Otro mpio.de B.C. & 10.3 & 12.0 & 10.3 & 24.9 & 5.4 \\
\hline Otro estado de México & 18.9 & 47.9 & 12.7 & 9.4 & 16.2 \\
\hline EstadosUnidos & 24.8 & 10.3 & 32.7 & 35.2 & 2.7 \\
\hline Destino no especificado & 0.8 & 0.0 & 0.0 & 0.0 & 5.4 \\
\hline
\end{tabular}

FUENTE: CONEPO-BC, IS-UABC. Encuesta continua de migración de B.C. 1987. Mexicali.

NOTA: La población emigrante corresponde a aquélla que abandonó la localidad durante los últimos 12 meses, por lo tanto, se consideran 21 meses efectivos de exposición. 


\section{LA MIGRACIÓN INTERURBANA EN BAJA CALIFORNIA}

\section{Volumen, saldos y temporalidad}

A partir del hecho de que $77.2 \%$ de los emigrantes a otro municipio de la entidad proceden de alguna de las cuatro localidades urbanas del estado, se asumió que al analizar el grupo de residentes urbanos inmigrantes (cuya residencia anterior fuese alguno de los cuatro municipios de Baja California) se estaría en posibilidad de suponer que se trata - por lo menos mayoritariamente- del grupo que integra los flujos migratorios interurbanos.

En relación a este grupo de migrantes intraestatales, la Encuesta Continua de Migración de B.C. permitió observar que su volumen es del orden de 68 mil personas. De ellas, el $62.6 \%$ se localiza en la ciudad de Tijuana; $20.3 \%$ en la ciudad de Ensenada; $10.1 \%$ en Mexicali y en la ciudad de Tecate radica en restante $7.0 \%$ de los inmigrantes intermunicipales (ver cuadro 7).

$\mathrm{Al}$ analizar la distribución de la residencia anterior de los migrantes intraestatales, destaca el hecho de que del total, la mitad proceden del municipio de Mexicali, el $18.9 \%$ provienen del municipio de Ensenada, el $16.6 \%$ del de Tecate, mientras que el municipio de Tijuana sólo aporta el $12.6 \%$ del total de los emigrantes hacia las ciudades del estado.

CUADRO 7. Migrantes intraestatales según origen y destino urbano. B.C. 1987.

\begin{tabular}{|c|c|c|}
\hline MUNICIPIO DE & & CIUDAD DE RESID. ACTUAL \\
\hline
\end{tabular}

$\begin{array}{lrrrrr}\text { TOTAL } & 67987 & 13803 & 6859 & 4770 & 42555 \\ \text { Ensenada } & 12853 & 0 & 2563 & 657 & 9633 \\ \text { Mexicali } & 34429 & 8022 & 0 & 2448 & 23959 \\ \text { Tecate } & 11252 & 968 & 2057 & 0 & 8227 \\ \text { Tijuana } & 8550 & 4736 & 2239 & 1575 & 0 \\ \text { N.E. } & 903 & 77 & 0 & 90 & 736\end{array}$

FUENTE: CONEPO-BC. Encuesta continua de migración de B.C. 1987. Mexicali,B.C., México. 
CUADRO 8. Saldos netos de la migración intraestatal. B.C. 1987.

\begin{tabular}{lrrrrr}
$\begin{array}{l}\text { MUNICIPIO DE } \\
\text { RESID. ANT. }\end{array}$ & B.C. TOTAL & CIUDAD DE RESID. ACTUAL \\
ENS. & MXL. & TEC. & TIJ. \\
\hline & & & & & \\
TOTAL & 0 & 873 & -27570 & -6572 & 33269 \\
Ensenada & 873 & 0 & -5459 & -311 & 4897 \\
Mexicali & -27570 & 5459 & 0 & 391 & 21720 \\
Tecate & -6572 & 311 & -391 & 0 & 6652 \\
Tijuana & 33269 & -4897 & -21720 & -6652 & 0
\end{tabular}

FUENTE: CONEPO-BC. Encuesta continua de migración de B.C. 1987. Mexicali, B.C., México.

Esta preponderancia del municipio de Mexicali como lugar de origen de la migración, se reproduce en las tres ciudades restantes del estado, pues en ellas más de la mitad de los inmigrantes proceden de dicho municipio. En el caso de Ensenada, el $58.1 \%$ de sus inmigrantes intraestatales provienen de Mexicali; en Tecate la proporción es del orden de $51.3 \%$, en tanto que en la ciudad de Tijuana el $56.3 \%$ de los inmigrantes intermunicipales se originan en Mexicali.

Ahora bien, observada en términos de los destinos que predominan en función del municipio de origen, destaca la capacidad de atracción de la ciudad de Tijuana, pues hacia ella se dirigen al menos dos tercios de todos los migrantes de los otros municipios del estado. Así, el $74.9 \%$ de los migrantes del municipio de Ensenada tiene como destino la ciudad de Tijuana; del total correspondiente a Mexicali $69.6 \%$ se dirige a esa localidad y de los migrantes de Tecate el $73.1 \%$ se localiza en Tijuana.

En este sentido, los rasgos arriba señalados permiten advertir un patrón de movilidad intraestatal, en el que los municipios de Mexicali y Tijuana adquieren el carácter de nodos polarizadores de las corrientes de migrantes, correspondiendo a Mexicali la función de área de expulsión de población y a la ciudad de Tijuana la de centro de recepción.

$\mathrm{Al}$ respecto, la información de los saldos migratorios que se presenta en el cuadro 8 permite constatar la predominancia del municipio y la ciudad mencionados. En el caso del municipio de Mexicali se observa que representa el $80.8 \%$ del saldo migratorio negativo total, puesto que esta ciudad aporta más emigrantes a las otras tres ciudades del estado 
que los inmigrantes que recibe de los tres municipios restantes; de ahí que su saldo migratorio resulte negativo en relación a los municipios de Ensenada, Tecate y Tijuana.

Por su parte, la ciudad de Tijuana presenta saldos migratorios positivos con todos los municipios restantes, lo que permite que agrupe al $97.5 \%$ del total del saldo positivo de migración intraestatal. El $2.5 \%$ remanente del saldo positivo lo absorbe la ciudad de Ensenada, la cual se caracteriza además por tener saldos del mismo signo con Mexicali y Tecate, en tanto que la ciudad de Tecate sólo genera saldos positivos con el municipio de Mexicali, convirtiéndose en la segunda área de expulsión neta de población en el estado.

Una vez establecida la magnitud y dirección de los movimientos intraestatales de población en Baja California, resulta conveniente analizar la distribución temporal con que se han presentado estos flujos de los residentes del estado. La primer característica que denota la estructura del

CUADRO 9. Inmigrantes intraestatales según tiempo de residencia. B.C. 1987.

\begin{tabular}{|c|c|c|c|c|c|}
\hline \multirow{2}{*}{$\begin{array}{l}\text { AÑOS DE } \\
\text { RESIDENCIA }\end{array}$} & \multirow[b]{2}{*}{ B.C. TOTAL } & \multicolumn{4}{|c|}{ CIUDAD DE RESID. ACTUAL } \\
\hline & & ENS. & MXL. & TEC. & TIJ. \\
\hline TOTAL & 67987 & 13803 & 6859 & 4770 & 42555 \\
\hline 10 menos & 7897 & 1032 & 1417 & 828 & 4620 \\
\hline 2 & 2148 & 226 & 928 & 198 & 796 \\
\hline 3 & 3725 & 1593 & 205 & 270 & 1657 \\
\hline 4 & 4191 & 202 & 188 & 180 & 3621 \\
\hline 5 & 1658 & 385 & 175 & 108 & 990 \\
\hline 6 & 2356 & 613 & 149 & 234 & 1360 \\
\hline 7 & 1853 & 227 & 1104 & 369 & 153 \\
\hline 8 & 1167 & 655 & 0 & 351 & 161 \\
\hline 9 & 1145 & 653 & 339 & 153 & 0 \\
\hline 10 & 1716 & 372 & 0 & 36 & 1308 \\
\hline 11 a 20 & 25223 & 4629 & 1288 & 1458 & 17848 \\
\hline Más de 20 & 14505 & 3216 & 1066 & 576 & 9647 \\
\hline N.E. & 403 & 0 & 0 & 9 & 394 \\
\hline
\end{tabular}

FUENTE: CONEPO-BC. Encuestacontinua de migración de B.C. 1987.Mexicali, B.C., México.

N.E. $=$ No especificado. 
tiempo de residencia de los inmigrantes de las áreas urbanas consiste en la alta concentración en un periodo relativamente reciente.

En efecto, como se puede observar en cl cuadro 9, virtualmente un tercio de todos los inmigrantes que residen en las ciudades de la entidad se movilizaron en el periodo comprendido de 1981 a 1987. En esos siete años, la ciudad de Ensenada recibio al 29.3\% de los inmigrantes que han arribado de otro municipio de Baja California, la ciudad de Mexicali al $44.6 \%$ de su total de inmigrantes intraestatales; en ese periodo, el $38.1 \%$ se localizo en Tecate, mientras que en Tijuana la proporción fue del orden de $30.7 \%$.

Observada a nivel agregado, el $41.0 \%$ de la emigración intraestatal con destino urbano se originó en los últimos diez afoos; el $37.1 \%$ en el periodo de 11 a 20 años y el $21.3 \%$ de los migrantes se relocalizaron en los municipios del estado hace más de 20 años. Esta estructura temporal de la migración intraestatal, indudablemente afectada por la incidencia de la mortalidad en los migrantes más antiguos, proporciona evidencia de un moderado pero reciente incremento de los flujos intermunicipales, cuyas características particulares se evalúan a continuación.

\section{Origen, edad, sexo, alfabetismo y actividad}

Al analizar al grupo de inmigrantes intraestatales que residen en las áreas urbanas de Baja California en función de su lugar de nacimiento, resulta factible distinguir tres tipos fundamentales de movimientos de población. El primero de ellos consiste en migraciones de retorno de nativos de la entidad, quienes regresan a su municipio de origen después de residir en otro municipio del estado. La segunda modalidad se integra por los nativos de Baja California que mudaron su residencia a otra ciudad del mismo estado pero en otro municipio. Por último, el tercer tipo de movimiento lo realizan los no-nativos de la entidad, en lo que aparece como migraciones por etapas.

En relación a estos tres tipos de movimientos, la información del cuadro 10 permite observar que más de la mitad $(53.1 \%)$ de ellos se integran por migrantes internos del país los cuales, después de residir en algún municipio de Baja California, mayoritariamente tienen como destino final la ciudad de Tijuana (64.1\% del total de dicho grupo). Al anterior le sigue en importancia el grupo de nativos de la entidad que han cambiado su municipio de residencia, pues representan al $42.9 \%$ del total de los migrantes intraestatales. Por su parte, la migración de retomo al municipio de origen agrupa al $3.0 \%$ del total de migrantes, y en ella participan prepon- 
CUADRO 10. Lugar de nacimiento y destino de los migrantes intraestatales.

\begin{tabular}{lrrrrr}
\hline $\begin{array}{l}\text { LUGAR DE } \\
\text { NAC. }\end{array}$ & B.C. TOTAL & \multicolumn{5}{c}{ CIUDAD DE RESID. ACTUAL } \\
ENS. & MXL. & TEC. & TIJ. \\
\hline TOTAL & 67987 & 13803 & 6859 & 4770 & 42555 \\
Este mpio. & 2020 & 124 & 868 & 72 & 956 \\
Otro mpio. & & & & & \\
de B.C. & 29179 & 6662 & 2001 & 2448 & 18068 \\
Otro estado & 36123 & 6880 & 3990 & 2106 & 23147 \\
Estados Unidos & 375 & 137 & 0 & 63 & 175 \\
Otro país & 290 & 0 & 0 & 81 & 209 \\
& & & & & \\
\hline
\end{tabular}

FUENTE: CONEPO-BC. Encuesta continua de migración de B.C.1987. Mexicali, B.C., México.

derantemente las ciudades de Tijuana y Mexicali. Por último, la migración por etapas de residentes nativos del extranjero representa sólo al $1.0 \%$ del total de los migrantes, observándose que ésta tiene como destinos finales predominantes a las ciudades de Ensenada y Tijuana.

En relación a la estructura por edad y sexo de la población migrante, la información que se presenta en los cuadros 11,12 y 13 permite identificar los siguientes rasgos característicos. La principal diferencia que se puede observar en la estructura de edad de la población total y la de la población migrante, radica en la reducida participación del grupo de edad de 0 a 14 años en el total de inmigrantes. En éstos, el grupo mencionado sólo representa al $9.1 \%$ del total, mientras que en el conjunto de la población de la entidad el mismo rango incluye al $35.3 \%$ de los habitantes del estado.

La desproporción que presenta ese primer grupo de edades en el caso de los migrantes, se compensa con la elevada participación que adquiere la población cuyas edades quedan comprendidas en los rangos de 15 a 39 y de 40 o más años de edad. En ellos, los inmigrantes representan el 59.3 y el $31.6 \%$ de su correspondiente total, en tanto que a nivel de la población total las proporciones de cada uno de estos grupos son del orden de $45.4 \mathrm{y}$ $19.3 \%$, respectivamente.

Sin embargo, las diferencias de edades observadas en el total de inmigrantes en relación al conjunto de la población no se presentan de manera consistente. Así, al analizar la estructura de los inmigrantes de cada 
CUADRO 11. Población total por grupos quinquenales de edad B.C. 1987.

\begin{tabular}{lrrrrr}
\hline $\begin{array}{l}\text { GRUPOS DE } \\
\text { EDAD }\end{array}$ & B.C. TOTAL & ENS. & MXL. & TEC. & TIJ. \\
\hline TOTAL & 1673460 & 212943 & 697519 & 39034 & 723964 \\
& & & & & \\
$0-4$ & 198707 & 23735 & 83773 & 4731 & 86468 \\
$5-9$ & 180665 & 26546 & 71543 & 4816 & 77760 \\
$10-14$ & 210756 & 27201 & 85835 & 5050 & 92670 \\
$15-19$ & 212160 & 24835 & 89764 & 4645 & 92916 \\
$20-24$ & 195705 & 21507 & 83883 & 3960 & 86355 \\
$25-29$ & 140849 & 15312 & 57994 & 3309 & 64234 \\
$30-34$ & 118708 & 16017 & 45100 & 2704 & 54887 \\
$35-39$ & 91523 & 14066 & 36238 & 2338 & 38881 \\
$40-44$ & 69673 & 10315 & 29951 & 1545 & 27862 \\
$45-49$ & 68208 & 7598 & 35283 & 1357 & 23970 \\
$50-54$ & 47139 & 7119 & 19169 & 1233 & 19618 \\
$55-59$ & 36658 & 4263 & 17249 & 922 & 14224 \\
$60-64$ & 38562 & 5029 & 17781 & 823 & 14929 \\
$65-$ más & 64147 & 9400 & 23956 & 1601 & 29190 \\
\hline
\end{tabular}

FUENTE: CONEPO-BC. IS-UABC. Encuesta continua de migración de B.C. 1987.

una de las cuatro ciudades del estado, destaca el hecho de que en las ciudades de Mexicali y Tecate la participación del grupo de 0 a 14 años se sitúa en 27.5 y $18.2 \%$ de su correspondiente total. Esta diferencia se reproduce de manera acentuada en el grupo de 40 años y más en el caso de Mexicali, donde la población inmigrante de esas edades sólo significa el $6.8 \%$ de su total.

El conjunto de rasgos diferenciales que presenta la estructura por edades de los inmigrantes, adquiere mayor relevancia al observar que éstos no cstán acompañados de una distribución por sexos que indique algún tipo de selectividad. Los valores que adquieren la proporción de hombres y mujeres, tanto en el total como en cada una de las ciudades, oscilan en torno al nivel de $50.0 \%$, con una variación máxima de 2.4 puntos porcentuales (ver cuadro 13).

Así, la relevancia de la estructura etaria de los inmigrantes, se pone de manifiesto al analizar el comportamiento de los indicadores referidos a la 
CUADRO 12. Inmigrantes urbanos intraestatales por grupos de edad.

\begin{tabular}{lrrrrr}
\hline $\begin{array}{l}\text { GRUPOS DE } \\
\text { EDAD }\end{array}$ & TOTAL & \multicolumn{5}{c}{ CIUDAD DE RESIDENCIA } \\
& URBANO & ENS. & MXL. & TEC. & \multicolumn{1}{c}{ TIJ. } \\
\hline TOTAL & 67987 & 13803 & 6859 & 4770 & 42555 \\
& & & & & \\
$0-4$ & 684 & 146 & 161 & 180 & 197 \\
$5-9$ & 3143 & 533 & 787 & 270 & 1553 \\
$10-14$ & 2396 & 553 & 939 & 414 & 490 \\
$15-19$ & 8685 & 1797 & 151 & 513 & 6224 \\
$20-24$ & 7707 & 1494 & 1322 & 567 & 4324 \\
$25-29$ & 7901 & 1006 & 992 & 621 & 5282 \\
$30-34$ & 8276 & 1859 & 1033 & 486 & 4898 \\
$35-39$ & 7720 & 1652 & 1003 & 396 & 4669 \\
$40-44$ & 4047 & 1074 & 0 & 261 & 2712 \\
$45-49$ & 2841 & 599 & 0 & 252 & 1990 \\
$50-54$ & 3332 & 1093 & 0 & 261 & 1978 \\
$55-59$ & 2646 & 432 & 161 & 180 & 1873 \\
$60-64$ & 3000 & 649 & 161 & 117 & 2073 \\
$65-$ más & 5609 & 916 & 149 & 252 & 4292 \\
\hline
\end{tabular}

FUENTE: CONEPO-BC, IIS-UABC. Encuesta continua de migración de B.C. 1987.

condición de alfabetismo y de actividad de los integrantes de este grupo. $\mathrm{Al}$ respecto se debe observar que, por una parte, los porcentajes de población alfabeta resultan virtualmente iguales en el conjunto estatal y en cada una de las ciudades al comparar la población urbana total con los inmigrantes de la ciudad correspondiente. Ello se explica, a juicio nuestro, por la menor desproporción que se observa entre los inmigrantes y la población urbana en términos de su composición por personas cuyas edades son superiores a los catorce años.

En la contraparte, el porcentaje de inmigrantes que actualmente asiste a la escuela resulta notoriamente inferior al correspondiente a la población urbana total, en concordancia con la reducida cantidad de personas de catorce anfos o menos de edad que integran el grupo de inmigrantes. A nivel de las cuatro ciudades, los porcentajes más altos de inmigrantes que asisten a 
CUADRO 13. Características generales de la población urbana e inmigrantes intraestatales.

\begin{tabular}{|c|c|c|c|c|c|c|c|c|c|c|}
\hline \multirow[t]{2}{*}{ Características } & \multicolumn{2}{|c|}{ Baja California } & \multicolumn{2}{|c|}{ Ensenada } & \multicolumn{2}{|c|}{ Mexicali } & \multicolumn{2}{|c|}{ Tecate } & \multicolumn{2}{|c|}{ Tijuana } \\
\hline & Urb. & Inm. & Urb. & Inm. & Urb. & Inm. & Urb. & Inm. & Urb. & Inm. \\
\hline$\%$ Hombres & 50.0 & 51.0 & 49.1 & 47.1 & 49.9 & 47.7 & 49.3 & 51.3 & 50.3 & 52.7 \\
\hline$\%$ Mujeres & 50.0 & 49.0 & 50.9 & 52.9 & 50.1 & 52.3 & 50.7 & 48.7 & 49.7 & 47.3 \\
\hline \% Pob.urbana & 100.0 & 5.0 & 100.0 & 9.0 & 100.0 & 1.4 & 100.0 & 16.1 & 100.0 & 6.2 \\
\hline \% Alfabetas & 94.5 & 93.7 & 95.5 & 95.5 & 94.9 & 95.1 & 94.7 & 96.0 & 93.9 & 92.6 \\
\hline $\begin{array}{l}\% \text { Asisten a la } \\
\text { escuela }\end{array}$ & 32.0 & 11.6 & 34.5 & 15.6 & 32.5 & 20.6 & 34.6 & 20.5 & 30.9 & 8.0 \\
\hline$\%$ Empleados & 47.9 & 56.7 & 47.9 & 53.3 & 46.5 & 50.4 & 48.8 & 53.1 & 48.8 & 59.0 \\
\hline$\%$ Desempleados & 4.3 & 2.2 & 2.9 & 2.2 & 4.7 & 0 & 4.8 & 2.3 & 4.4 & 2.3 \\
\hline $\begin{array}{l}\text { Tasa de } \\
\text { particip. masc. }\end{array}$ & 69.2 & 85.5 & 69.3 & 76.8 & 68.8 & 100.0 & 71.4 & 78.5 & 69.3 & 87.0 \\
\hline $\begin{array}{l}\text { Tasa de } \\
\text { particip. fem. }\end{array}$ & 28.3 & 25.7 & 28.0 & 32.2 & 24.8 & 11.6 & 27.2 & 27.4 & 29.1 & 25.4 \\
\hline
\end{tabular}

FUENTE: CONEPO-BC. Encuesta continua de migración de B.C. 1987. Mexicali, B.C. México. 
la escuela coinciden con las mayores proporciones de inmigrantes en edades jóvenes en Mexicali y Tecate, mientras que en Ensenada y Tijuana se observa una asistencia inferior a la de la población total.

Otro rubro en el que la composición por edades de los inmigrantes aparece como determinante es el relativo a la condición de actividad económica. En este caso, tres indicadores resultan consistentemente favorables para la población inmigrante. Así, el porcentaje de población en edad de trabajar ocupada es mayor para los inmigrantes; la tasa de desempleo de estos últimos resulta menor que la del conjunto de la población urbana total, y la tasa bruta de participación masculina de los inmigrantes es mayor que la de su contraparte urbana.

Las dos últimas características, relativas a la asistencia a servicios de educación y a la participación en las actividades económicas, dan pie para intentar un esbozo de los rasgos que presenta el perfil educativo y laboral de este grupo de inmigrantes intraestatales, tarea que se realiza en el apartado que se presenta a continuación.

\section{Escolaridad y ocupación}

Al analizar las características del último nivel de escolaridad, de posición en el empleo y de rama de actividad de los inmigrantes intraestatales se optó por realizar una comparación entre la estructura proporcional que presenta la población urbana total y la correspondiente a la población inmigrante de cada una de las ciudades del estadc, esto con el propósito de identificar los rasgos diferenciales que presenta el último grupo en cada uno de los tres indicadores seleccionados.

Con base en la información del cuadro 14 y a través del procedimiento mencionado, resulta posible observar que, en materia de niveles de escolaridad, la población inmigrante intraestatal presenta un perfil en el que cinco niveles escolares cuentan con una mayor proporción que la correspondiente a la población urbana total.

Un rasgo que acompaña a estos cinco niveles de escolaridad consiste en su agrupamiento en los extremos de la secuencia de la educación formal. Expuesto en otros términos, lo anterior implica que los inmigrantes presentan una estructura con participaciones comparativamente desproporcionadas en los grupos de población sin estudios, en los de técnicos de secundaria, en los de profesores normalistas, en los de profesionistas y en los de personas con posgrados.

Este agrupamiento desproporcionado en los extremos de los niveles educativos que presenta el conjunto de la población inmigrante se reproduce a nivel de los inmigrantes de las ciudades del estado, con la única 
CUADRO 14. Población urbana e inmigrantes intraestatales según escolaridad.

\begin{tabular}{|c|c|c|c|c|c|c|c|c|c|c|}
\hline \multirow[t]{2}{*}{ Escolaridad } & \multicolumn{2}{|c|}{ BAJA CALIF. } & \multicolumn{2}{|c|}{ ENSENADA } & \multicolumn{2}{|c|}{ MEXICALI } & \multicolumn{2}{|c|}{ TECATE } & \multicolumn{2}{|c|}{ TIJUANA } \\
\hline & URB. & INM. & URB. & INM. & URB. & INM. & URB. & INM. & URB. & INM. \\
\hline TOTAL & 165422 & 66893 & 132093 & 13520 & 414201 & 6546 & 25155 & 4518 & 593973 & 42309 \\
\hline Sin estudios & 77521 & 7625 & 7300 & 980 & 22168 & 161 & 1638 & 369 & 46415 & 6115 \\
\hline Jardín de niños & 26573 & 933 & 3408 & 51 & 10358 & 161 & 558 & 27 & 12249 & 694 \\
\hline Primaria & 533834 & 29899 & 57601 & 5688 & 167403 & 2908 & 12033 & 1980 & 296797 & 19323 \\
\hline Téc.con prim. & 7770 & 367 & 1743 & 138 & 3960 & 184 & 126 & 45 & 1941 & 0 \\
\hline secundaria & 265050 & 9200 & 28088 & 2333 & 107557 & 945 & 5922 & 945 & 123483 & 4977 \\
\hline Téc. con sec. & 42112 & 2639 & 8832 & 1313 & 19166 & 152 & 612 & 135 & 13502 & 1039 \\
\hline Preparatoria & 98786 & 4465 & 9123 & 742 & 37993 & 933 & 2151 & 441 & 49519 & 2349 \\
\hline $\begin{array}{l}\text { Téc. con } \\
\text { preparatoria }\end{array}$ & 14773 & 804 & 2318 & 257 & 7105 & 152 & 333 & 108 & 5017 & 287 \\
\hline Normal & 21394 & 4487 & 2956 & 460 & 6829 & 205 & 297 & 144 & 11312 & 3678 \\
\hline Profesional & 72437 & 6183 & 9987 & 1512 & 29531 & 745 & 1368 & 288 & 31551 & 3638 \\
\hline Posgrado & 3457 & 273 & 426 & 46 & 1159 & 0 & 54 & 18 & 1818 & 209 \\
\hline No especificado & 1715 & 18 & 311 & 0 & 972 & 0 & 63 & 18 & 369 & 0 \\
\hline
\end{tabular}

FUENTE: CONEPO-BC. Encuesta continua de migración de B.C. 1987. Mexicali. 
CUADRO 15. Población urbana e inmigrantes intraestatales según posición de empleo.

\begin{tabular}{|c|c|c|c|c|c|c|c|c|c|c|}
\hline POSICIÓN & BA J & CALIF. & ENSE & NADA & MEXI & CALI & TEC & TE & TนJU & NA \\
\hline DE EMPLEO & URB. & INM. & URB. & INM. & URB. & INM. & URB. & INM. & URB. & INM. \\
\hline TOTAL & 476370 & 35853 & 53222 & 6923 & 166620 & 2746 & 10161 & 2205 & 246367 & 23979 \\
\hline $\begin{array}{l}\text { Patrón o } \\
\text { empleador }\end{array}$ & 8874 & 283 & 1755 & 175 & 1555 & 0 & 360 & 108 & 5204 & 0 \\
\hline Subcontrat. & 767 & 67 & 67 & 67 & 483 & 0 & 45 & 0 & 172 & 0 \\
\hline Auto empleo & 77752 & 7274 & 9860 & 1393 & 29482 & 482 & 1116 & 225 & 37294 & 5174 \\
\hline $\begin{array}{l}\text { Miembro de } \\
\text { cooperativa }\end{array}$ & 1683 & 65 & 1405 & 65 & 278 & 0 & 0 & 0 & 0 & 0 \\
\hline Empleo fijo & 321422 & 20296 & 33109 & 4157 & 121771 & 1990 & 7407 & 1584 & 159135 & 12565 \\
\hline A destajo & 51753 & 4448 & 5403 & 766 & 9042 & 113 & 1044 & 279 & 36264 & 3290 \\
\hline Sin salario & 55 & 46 & 46 & 46 & 0 & 0 & 9 & 0 & 0 & 0 \\
\hline $\begin{array}{l}\text { Familiar sin } \\
\text { salario }\end{array}$ & 11187 & 3365 & 1198 & 254 & 3003 & 161 & 81 & 0 & 6905 & 2950 \\
\hline Otros & 723 & 0 & 108 & 0 & 0 & 0 & 9 & 0 & 606 & 0 \\
\hline N.E. & 2154 & 9 & 271 & 0 & 1006 & 0 & 90 & 9 & 787 & 0 \\
\hline
\end{tabular}

FUENTE : CONEPO-BC. Encuesta continua de migración de B.C. 1987. Mexicali, B.C. México.

N.E. $=$ No especificado. 
CUADRO 16. Población urbana e inmigrante intraestatal por tipo de actividad.

\begin{tabular}{|c|c|c|c|c|c|c|c|c|c|c|}
\hline \multirow{2}{*}{$\begin{array}{l}\text { TIPO DE } \\
\text { ACTIVIDAD } \\
\end{array}$} & \multicolumn{2}{|c|}{ BAJA CALIF. } & \multicolumn{2}{|c|}{ ENSENADA } & \multicolumn{2}{|c|}{ MEXICALI } & \multicolumn{2}{|c|}{ TECATE } & \multicolumn{2}{|c|}{ TIJUANA } \\
\hline & URB. & INM. & URB. & INM. & URB. & INM. & URB & INM. & URB. & INM. \\
\hline TOTAL & 476370 & 35853 & 53222 & 6923 & 166620 & 2746 & 10161 & 2205 & 246367 & 23979 \\
\hline Agricultura & 25521 & 5769 & 1179 & 88 & 9031 & 0 & 279 & 81 & 15032 & 5600 \\
\hline Pesca & 4483 & 441 & 4305 & 441 & 0 & 0 & 9 & 0 & 169 & 0 \\
\hline Minería & 586 & 0 & 152 & 0 & 145 & 0 & 18 & 0 & 271 & 0 \\
\hline Ind. transf. & 54919 & 2368 & 2588 & 240 & 23170 & 313 & 1719 & 378 & 27442 & 1437 \\
\hline Ind.de ensam. & 37848 & 1442 & 808 & 124 & 8272 & 0 & 1521 & 297 & 27247 & 1021 \\
\hline Construcción & 39123 & 1233 & 3912 & 544 & 16852 & 0 & 981 & 252 & 17378 & 437 \\
\hline Electricidad & 5586 & 27 & 312 & 0 & 3590 & 0 & 207 & 27 & 1477 & 0 \\
\hline Comercio & 99419 & 5344 & 14986 & 1945 & 41270 & 632 & 1539 & 288 & 41624 & 2479 \\
\hline Transporte & 20350 & 587 & 2101 & 156 & 6944 & 198 & 324 & 36 & 10981 & 197 \\
\hline Gobierno & 44985 & 4008 & 8718 & 1594 & 22255 & 274 & 972 & 225 & 13040 & 1915 \\
\hline Seg. $y$ finanzas & 2410 & 240 & 219 & 0 & 1555 & 0 & 108 & 27 & 528 & 213 \\
\hline Turismo & 21953 & 2169 & 1768 & 338 & 3491 & 0 & 369 & 144 & 16325 & 1687 \\
\hline Educación & 20682 & 1827 & 2135 & 314 & 10987 & 205 & 342 & 108 & 7218 & 1200 \\
\hline Serv. domést. & 12587 & 947 & 1342 & 162 & 3015 & 0 & 207 & 54 & 8023 & 731 \\
\hline Serv.tec./prof. & 43745 & 3607 & 6602 & 596 & 12044 & 572 & 612 & 135 & 24487 & 2304 \\
\hline Otros serv. & 32361 & 3075 & 1265 & 177 & 2272 & 552 & 810 & 144 & 28014 & 2202 \\
\hline I.E. & 6083 & 2610 & 54 & 54 & 274 & 0 & 54 & 0 & 5701 & 2556 \\
\hline N.E. & 3729 & 159 & 776 & 150 & 1453 & 0 & 90 & 9 & 1410 & 0 \\
\hline
\end{tabular}

FUENTE: CONEPO-BC. Encuesta continua de migración de B.C. 1987. Mexicali, B.C., México.

I.E. $=$ Insuficientemente especificado.

N.E. $=$ No especificado. 
excepción de la ciudad de Mexicali, donde se observa que las proporciones de inmigrantes sin estudios y de los que cuentan con posgrado son inferiores a los de la población total de esa ciudad.

Analizada en esta misma perspectiva, la información correspondiente a la posición en el empleo (cuadro 15), indica que la población inmigrante presenta una estructura predominantemente concentrada en las categorías de trabajadores por su cuenta, trabajadores a sueldo fijo, trabajadores a destajo y trabajadores familiares sin pago. Sin embargo, comparativamente con la estructura ocupacional del total de la población urbana, los inmigrantes presentan participaciones desproporcionadas en las categorías de subcontratistas, trabajadores por su cuenta, trabajadores a destajo, trabajadores no familiares sin pago y trabajadores familiares sin pago.

Siguiendo con este enfoque de análisis, por último, se debe advertir que al igual que en el caso del nivel de escolaridad, la estructura por rama de actividad de los inmigrantes presenta una marcada especialización. En este caso, a partir de la información del cuadro 16, se puede observar que los inmigrantes participan desproporcionadamente en las ramas de actividades agropecuarias, pesca, gobierno, seguros y finanzas, turismo, educación, servicios profesionales o técnicos y otros servicios, en comparación a la estructura que presenta el total de la población urbana.

Esta concentración del total de la población inmigrante en los sectores primario y de servicios se reproduce con variaciones mínimas en la estructura de actividades de los inmigrantes de las ciudades del estado. A este nivel, las estructuras de las ciudades de Ensenada y Mexicali son las que presentan una mayor divergencia, pues en ellas se nota la ausencia del predominio de los inmigrantes en las actividades agropecuarias, así como una menor participación de las ramas del sector servicios en la última ciudad mencionada.

\section{CONCLUSIONES}

A partir de lo hasta aquí expuesto, resulta conveniente puntualizar algunos de los elementos que consideramos de mayor relevancia para los fines de este documento. En orden de presentación, los puntos a destacar son los siguientes:

a) La frontera norte de México, con los seis estados que la integran, ha mostrado una dinámica demográfica que le ha permitido aumentar su participación en la población nacional. En este proceso, la subregión integrada por los estados de Sonora y Baja California ha mostrado un mayor dinamismo, a la vez que sus municipios colindantes con la frontera internacional han concentrado una mayor cantidad de población residente en esos estados. 
b) A nivel de la frontera norte de México, la inmigración ha jugado un papel determinante en su dinámica demográfica de las últimas cinco décadas, y en el caso específico del estado de Baja California, a este factor se ha agregado el carácter acelerado y reciente de su proceso de poblamiento.

c) En ese contexto de acelerado y reciente poblamiento de Baja California, durante 1987 se logro estimar que la emigración en el estado es del orden del $1.6 \%$ anual; que su origen es urbano en $81.9 \%$ de los casos; que para el $8.2 \%$ de los inmigrantes el destino del movimiento es otro de los municipios del estado y que tres de cada cuatro migrantes intermunicipales proceden de las áreas urbanas de la entidad.

d) Para analizar estos movimientos intermunicipales con destino urbano, se seleccion 6 a los residentes de las ciudades de Baja California que declararon como lugar de residencia anterior a alguno de los municipios del estado. Para 1987, este grupo se estimó en aproximadamente $68 \mathrm{mil}$ personas, de las cuales el $50.0 \%$ procedían del municipio de Mexicali y el $62.0 \%$ residian en la ciudad de Tijuana.

e) Este grupo de inmigrantes intermunicipales que residen $\mathrm{cn}$ las ciudades de Baja California, se integra a partir de tres tipos fundamentales de movimientos migratorios: los nativos que retornan al municipio de origen, los nativos de la entidad que cambian de municipio de residencia $\mathrm{y}$ los no nativos que migran por etapas hacia y desde un municipio del estado.

f) En términos generales, las principales características de este grupo de población inmigrante consisten en que: un tercio de los movimientos se generaron entre 1981 y 1987; las edades mayores a los 14 años concentran a la mayor proporción de los integrantes sin mostrar composiciones diferenciadas por sexo; su nivel de alfabetismo es similar al de la población urbana total, pero su proporción de asistentes a los servicios educativos es menor; su porcentaje de población ocupada es superior a la del total de los habitantes de las ciudades, a la vez que sus tasas de desempleo resultan menores que las de estos últimos y sus estructuras por nivel educativo y por rama de actividad económica muestran distribuciones polarizadas, donde predominan las personas sin estudios y las que han alcanzado cl nivel superior por una parte, así como la inserción en los sectores primario y terciario de la actividad productiva, por la otra parte.

Este conjunto de características de los migrantes intermunicipales que residen en las ciudades de Baja California, nos permiten elaborar algunas conclusiones en relación a la migración urbana-urbana que se genera en el estado. La primera de ellas consiste en que efectivamente este movimiento intraestatal, aunque de factura añeja, adquiere mayor dinamismo 
en fechas recientes, tal como lo señalan los datos correspondientes al tiempo de residencia de los inmigrantes.

Un segundo elemento de relevancia estatal se integra a partir del hecho de que la mitad de la migración se origina en el municipio de Mexicali. En este sentido, se define una redistribución de la población de la entidad, en lo que aparece como un movimiento desde al área del valle del Río Colorado hacia la zona de la costa del Pacífico.

Relacionado con el anterior, un tercer elemento de importancia regional radica en los saldos migratorios positivos que generan las ciudades de Ensenada y Tijuana, pues en conjunto estructuran un corredor de atracción a la inmigración de población del estado, en un desplazamiento que favorece la ubicación en el extremo noroeste de la península de Baja California.

Las características de edad, escolaridad y participación productiva del grupo migrante, en su conjunto nos llevan a concluir que se está en presencia de movimientos fundamentalmente originados por motivos laborales. Los integrantes de estos movimientos se perfilan como personas en edades productivas, con niveles elevados de escolaridad y con predominancia a ubicarse en el sector de servicios de las economías de las ciudades de destino.

Con estos elementos en mente, resulta posible advertir que los movimientos de migración urbana-urbana en Baja California presentan ciertas particularidades que implícitamente determinan el tipo de políticas de desarrollo urbano que requieren implementarse en sus ciudades medias. Por exclusión, la principal determinante radica en la reducida presión que estos movimientos ejercen sobre la infraestructura y los servicios educativos de las áreas de recepción, pues como se ha mencionado, la población migrante presenta un perfil educativo elevado y aparentemente consolidado. Sin embargo, en la contraparte se observa un conjunto de factores a tomar en cuenta al diseffar las acciones que se relacionan con la demanda efectiva que acompafia al grupo inmigrante, pues con ella se generan presiones sobre la oferta de vivienda y sus servicios conexos, sobre la infraestructura y equipamiento para el abasto y la distribución de bienes de consumo; sobre los servicios de comunicación y transporte, sobre el equipamiento para la recreación y el deporte, y sobre la capacidad de la planta productiva de estas ciudades de Baja California. 